\title{
Phase-locking swallows in coupled oscillators with delayed feedback
}

\author{
Oleksandr V. Popovych, ${ }^{1}$ Valerii Krachkovskyi, ${ }^{1}$ and Peter A. Tass ${ }^{1,2}$ \\ ${ }^{1}$ Institute of Neuroscience and Medicine-Neuromodulation (INM-7), Research Center Jülich, 52425 Jülich, Germany \\ ${ }^{2}$ Department of Stereotaxic and Functional Neurosurgery, University Hospital, 50924 Cologne, Germany
}

(Received 11 June 2010; published 4 October 2010)

\begin{abstract}
We show that a nonlinear coupling with delayed feedback between two limit-cycle oscillators can lead to phase-locked, periodically modulated, and chaotic phase synchronization as well as to desynchronization. Parameter regions with stable phase-locked states attain the well-known form of the swallows or shrimps found and studied for nonlinear maps. We demonstrate that the swallow regions can be accompanied by a different bifurcation scenario where the periodic orbits of the phase-locked states undergo a torus bifurcation instead of a previously reported period-doubling bifurcation. This property has an impact on the spatial organization of the swallows in the parameter space. The swallow regions contribute to the synchronization domain of the considered system, and we analytically approximate the parameter synchronization threshold.
\end{abstract}

DOI: 10.1103/PhysRevE.82.046203

PACS number(s): 05.45.Xt

\section{INTRODUCTION}

If the coupling strength among elements of oscillatory ensembles increases, the oscillators adjust their individual frequencies and get synchronized [1-3]. Synchronization is a well-known phenomenon observed in many model systems ranging from the neurosciences and biology [4-7] to physics, chemistry, and engineering [8-10]. Since in real systems the communication among interacting functional units inevitably takes place with some delay in time because of the finite speed of signal propagation, it has been taken into account for modeling complex dynamical processes in coupled lasers $[11,12]$, electrochemical oscillators [13], neural networks [14-17], biological oscillators $[18,19]$, and neuronal systems $[20,21]$.

Retarded interactions between oscillators have been shown to significantly influence the collective dynamics of the interacting ensembles leading either to suppression or facilitation of synchronization $[11,12,14,22,23]$. Nevertheless, although synchronization of the oscillators coupled with delay could be disrupted for small and intermediate coupling strength if compared to the delay-free case, they eventually synchronize as the coupling strength gets large enough $[22,23]$. The time delay in coupling generally leads to the emergence of multistability of synchronized states [24-26] phase locked at different frequencies, where the number of coexisting stable phase-locked regimes grows proportionally to the coupling strength [27].

The situation is different if the delay is introduced to the coupling in the form of a delayed self-feedback [28,29]. For such a system the increasing coupling strength has a twofold impact on the synchronized dynamics of the oscillators: they first mutually phase lock to each other and then desynchronize via a complicated bifurcation transition as the coupling strength further increases. This property induced by the time delay has been used to develop a nonlinear delayed feedback method for the control of undesirable neuronal synchronization which is characteristic for, e.g., Parkinson disease or essential tremor [30-33].

In this paper, we consider a system of two oscillators nonlinearly coupled with delayed feedback. We focus on the bifurcations of the phase-locked states as parameters vary and show that their stability regions in the parameter space have an interesting form resembling much those of stable periodic orbits found for one- and two-dimensional nonlinear maps $[34,35]$. In the literature such stability regions are known as swallows, shrimps, or crossroad areas [36-38]. They demonstrate a universal character and have also been found and studied for periodically driven dissipative nonlinear oscillators [39], loss-modulated $\mathrm{CO}_{2}$ lasers [40], parametrically excited systems [41], Rössler oscillator [42], Belousov-Zhabotinsky reaction [43], and mechanical vibroimpact system [44]. The swallowlike structures have also been found within the Arnol'd tongues describing parameter regions of stable phase locking $[6,45,46]$.

The discussed swallow regions share the common property that at least one of their borders in the parameter plane is a curve of a period-doubling bifurcation. This property leads to a remarkable hierarchical structure in the parameter space, which is composed of swallows concatenated along the curves of the period-doubling bifurcations $[6,37,47,48]$. In such a way, for a proper choice of a path in the parameter space, a cascade of period-doubling bifurcations can be observed $[35,41,47]$. The other border of a swallow region can either be a curve of a period-doubling or a saddle-node bifurcation $[37,38]$. The latter case is realized if the swallow is the main stability region where the corresponding stable periodic orbit emerges via a saddle-node bifurcation giving rise to the above nesting structure of the swallows.

This paper argues that the course of bifurcations associated with the swallow regions can have a different scenario. We show that a stable periodic orbit may undergo no period doubling when the parameter point leaves the swallow stability region, but a torus bifurcation. This leads to a different organization of the swallows in the parameter space, which then do not form any hierarchical cluster of swallows, but remain stand-alone objects. In the considered model, these parameter regions are centered at the values of the time delay $\tau=n T / 2$, where $T$ is the mean natural period of the system, and their size decays inversely proportionally to the value of delay as the latter increases. 


\section{COUPLED OSCILLATORS WITH DELAYED FEEDBACK}

A simple model of two delay-coupled phase oscillators has been considered by Schuster and Wagner [24], where the signal from one oscillator to the other one is transmitted with a finite time delay. Multistability of many different phaselocked states, which emerge and remain stable with increasing coupling strength, has been revealed. In this paper we consider a system of two interacting phase oscillators with a different form of coupling. The signal from one oscillator to the other is transmitted instantaneously (or the corresponding delay can be neglected), whereas the self-feedback signal comes with a finite time delay:

$$
\left\{\begin{array}{l}
\dot{\psi}_{1}(t)=\omega_{1}+K \sin \left[\psi_{2}(t)-\psi_{1}(t-\tau)\right], \\
\dot{\psi}_{2}(t)=\omega_{2}+K \sin \left[\psi_{1}(t)-\psi_{2}(t-\tau)\right] .
\end{array}\right.
$$

$\psi_{1}$ and $\psi_{2}$ are the phases of the oscillators, $\omega_{1}$ and $\omega_{2}$ are their natural frequencies, $K$ is the coupling strength, and $\tau$ is the time delay. The system of two coupled phase oscillators (1) models the phase dynamics of two limit-cycle oscillators nonlinearly coupled with delayed feedback,

$$
\left\{\begin{array}{l}
\dot{z}_{1}=\left(a_{1}+i \omega_{1}-\left|z_{1}\right|^{2}\right) z_{1}+K z_{1}(t) z_{2}(t) z_{1}^{*}(t-\tau), \\
\dot{z}_{2}=\left(a_{2}+i \omega_{2}-\left|z_{2}\right|^{2}\right) z_{2}+K z_{2}(t) z_{1}(t) z_{2}^{*}(t-\tau) .
\end{array} .\right.
$$

$z_{j}(t), j=1,2$, are complex variables which, in the absence of coupling $(K=0)$, uniformly rotate with the natural frequencies $\omega_{j}$ on circles with radii $\sqrt{a_{j}}$. The asterisk denotes the complex conjugacy. Representing the complex variables in the form $z_{j}(t)=r_{j}(t) \exp \left(i \psi_{j}(t)\right)$, we can rewrite system (2) in the amplitudes $r_{j}$ and phases $\psi_{j}, j=1,2$. Assuming that the amplitudes $r_{j}(t)$ remain constant or close to that, one can neglect the amplitude dynamics and keep the phase equations only. The latter then attain the form (1).

The above assumption on the amplitude dynamics is not always fulfilled, and the amplitudes play an essential role in the dynamics of system (2). Nevertheless, the system of two coupled phase oscillators (1) reflects the main dynamical features of the two coupled limit-cycle oscillators (2). In particular, the dynamics of the phase-locked states of the limitcycle oscillators (2) is well approximated by the phase oscillators (1). Indeed, in this regime the phases can be represented as $\psi_{1,2}(t)=\Omega t \pm \alpha / 2$, and the phase difference $\varphi$ $=\psi_{2}-\psi_{1}=\alpha$ is constant [24], which then leads to constant amplitudes and makes the above assumption valid. Furthermore, exemplary one-parameter bifurcation diagrams of systems (1) [Fig. 1(a)] and (2) [Figs. 1(b) and 1(c)] demonstrate remarkable similarities.

As the coupling strength increases, oscillators (2) and (1) synchronize, where a stable phase-locked state emerges at $K=K_{s n}$ [Figs. 1(a) and 1(b)]. The phase difference $\varphi$ gets constant, and, as mentioned above, the same holds for the amplitudes $r_{j}$ [Fig. 1(c)]. The phase-locked dynamics is stable within a range of the coupling strength $K \in\left(K_{s n}, K_{t}\right)$. If parameter $K$ exceeds the upper bound of this interval, the phase difference starts to periodically oscillate, which again takes place for both systems (1) and (2) [Figs. 1(a) and 1(b)]. In the course of a further increase of the coupling strength
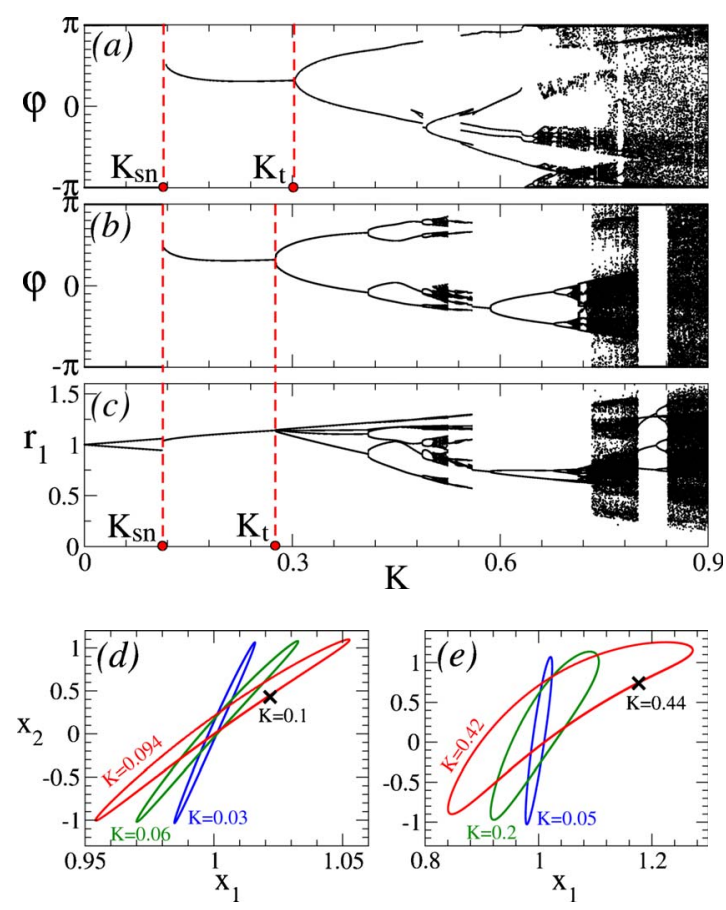

FIG. 1. (Color online) Attracting states of (a) system (1) and (b), (c) system (2) are depicted in the phase difference variable $\varphi=\psi_{2}$ $-\psi_{1}$ taken $(\bmod 2 \pi)$ in (a) and (b) and the amplitude $r_{1}$ in (c) versus the coupling parameter $K$. The stable steady states and the local maxima and minima of the trajectories $\varphi(t)$ and $r_{1}(t)$ are plotted after a skipped transient. The vertical dashed lines bound the parameter region of a stable phase-locked state. $K_{s n}$ and $K_{t}$ denote parameter $K$ values of the onset and loss of the phase-locked synchronization. (d), (e) Dynamics of the delay-free $(\tau=0)$ limit-cycle oscillators (2) $z_{j}=x_{j}+i y_{j}, j=1,2$, in the Poincaré section $\left\{x_{1} \geq 0, y_{1}=0\right\}$ shown in the plane $\left(x_{1}, x_{2}\right)$ for two different frequency mismatches $\Delta_{1}=\omega_{2}-\omega_{1}=0.2$ in (d) and $\Delta_{1}=1$ in (e). The closed curves correspond to a quasiperiodic dynamics, and the crosses indicate phase-locked synchronized states. The corresponding values of the coupling strength $K$ are shown. Parameters $\tau=4.0$ in (a)-(c), $\tau=0$ in (d) and (e), $\omega_{1}=2.9$ and $\omega_{2}=3.1$ in (a) $-(\mathrm{d})$, $\omega_{1}=2.5$ and $\omega_{2}=3.5$ in (e), and $a_{1}=1$ and $a_{2}=1.1$ in (a)-(e).

the phase differences of the limit-cycle and phase oscillators follow complicated sequences of bifurcations, which are somewhat different for the two systems. The amplitude dynamics of system (2) is apparently involved in it. However, for large coupling the phase difference $\varphi$ gets unbounded and, taken $(\bmod 2 \pi)$, fills the entire interval $(-\pi, \pi)$, which indicates an onset of desynchronization for the phase as well as for the limit-cycle oscillators [Figs. 1(a) and 1(b)]. In this sense the phase dynamics of the two nonlinearly coupled limit-cycle oscillators with delayed feedback (2) is well approximated by the system of phase oscillators (1).

We note that a relatively small frequency detuning $\Delta_{1}=\omega_{2}-\omega_{1}=0.2$ will be considered below. It is known that, even without delay, a large frequency detuning may lead to a variety of transitions to synchronization including oscillation death and suppression of the natural dynamics [2,3], where the amplitudes of coupled oscillators play an essential role. The considered value of $\Delta_{1}$ belongs to a range of the fre- 

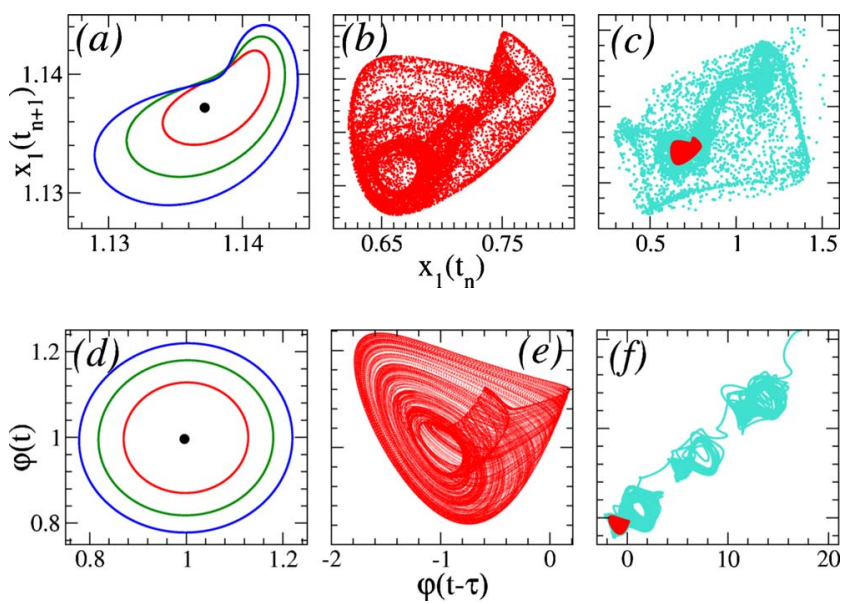

FIG. 2. (Color online) Amplitude and phase dynamics of the limit-cycle oscillators (2). (a)-(c) Dynamics of the first oscillator $z_{1}=x_{1}+i y_{1}$ in the Poincaré section $\left\{x_{1} \geq 0, y_{1}=0\right\}$ shown in the plane $\left(x_{1}\left(t_{n}\right), x_{1}\left(t_{n+1}\right)\right)$, where $t_{n}, n=0,1,2, \ldots$ are the times of the recurrent crossing of the Poincaré section by the trajectory $\left(x_{1}(t), y_{1}(t)\right)$. (d)-(f) Dynamics of the phase difference $\varphi$ shown in the $(\varphi(t-\tau), \varphi(t))$ plane. Coupling strength (a),(d) $K=0.27$ (black filled circle), $K=0.275$ (most inner red curve), $K=0.276$ (middle green curve), $K=0.277$ (most outer blue curve), (b), (e) $K=0.73$ (dark red dots), and (c), (f) $K=0.75$ (light blue dots). Attracting states in (c) and (f) (light blue dots) are overlapped with those from plots (b) and (e) (dark red dots), respectively, for comparison. Parameters $\tau=4.0, \omega_{1}=2.9, \omega_{2}=3.1, a_{1}=1$, and $a_{2}=1.1$.

quency mismatch where a phase-locked synchronized state emerges in system (2) via a saddle-node bifurcation of limit cycles on a two-dimensional torus as the coupling strength increases [Figs. 1(d) and 1(e)]. The coupled phase oscillators (1) without delay also demonstrate this mechanism of synchronization onset.

The loss of the phase-locked synchronization by oscillators (2), where they oscillate with constant phase shift $\varphi$ and constant amplitudes $r_{j}$, takes place at $K=K_{t}$. In the Poincaré section this transition corresponds to the emergence of an invariant closed curve from a fixed point [Fig. 2(a)]. At this transition a stable limit cycle thus bifurcates into a stable two-dimensional torus. After the bifurcation the amplitude is no longer constant, but oscillates periodically [Fig. 1(c)]. The same holds for the phase difference $\varphi$, where the torus bifurcation at $K=K_{t}$ leads to periodic oscillations of $\varphi$ [Figs. 2(d) and 1(b)]. The amplitude and phase oscillatory dynamics can develop to a chaotic behavior as the coupling strength between the oscillators increases. Examples of chaotic attractors for the amplitude and phase dynamics of the limit-cycle oscillators (2) are illustrated in Fig. 2(b) and 2(e), respectively. In spite of the chaotic dynamics, the phase difference $\varphi$ remains bounded, and, therefore, a regime of chaotic phase synchronization is established in system (2). The chaotic attractor then undergoes a crisis bifurcation and suddenly increases in size for larger coupling strength [Fig. 2(c)]. The phase difference starts to exhibit unbounded growth [Fig. 2(f)]. This indicates an onset of desynchronization in system (2).

Summarizing at this point, we note that the nonlinear coupling with delayed feedback in the form (2) has a twofold effect on the dynamics of the coupled oscillators. On the one hand, the coupling between the oscillators synchronizes them, and a stable phase-locked regime emerges for an intermediate range of the coupling strength. On the other hand, if the coupling strength gets large, the phase-locked state loses its stability via a torus bifurcation giving birth to a stable two-dimensional torus and to a state of periodically modulated phase synchronization with periodically oscillating and bounded phase difference. Finally, a chaotic phase synchronization emerges. The corresponding chaotic attractor then undergoes a crisis bifurcation at a larger coupling strength, and the strongly coupled oscillators desynchronize. Such synchronization-desynchronization transitions take place in the system of coupled limit-cycle oscillators (2) as well as in the system of coupled phase oscillators (1) [28,29]. The main dynamical regimes of the coupled limit-cycle oscillators are thus governed by their phase dynamics and are well reflected by the simple phase model (1), where the amplitude dynamics is ignored. In what follows we focus on the dynamics of system (1) and study the bifurcation mechanism of the emergence and destabilization of the phase-locked synchronized states.

\section{PHASE-LOCKED STATES}

We consider system (1) in variables of the phase difference $\varphi=\psi_{2}-\psi_{1}$ and the mean phase $\phi=\left(\psi_{2}+\psi_{1}\right) / 2$

$$
\left\{\begin{array}{l}
\dot{\varphi}(t)=\Delta_{1}-2 K \sin \left[\frac{\varphi(t)+\varphi(t-\tau)}{2}\right] \times \cos [\phi(t)-\phi(t-\tau)], \\
\dot{\phi}(t)=\Delta_{2}+K \cos \left[\frac{\varphi(t)+\varphi(t-\tau)}{2}\right] \times \sin [\phi(t)-\phi(t-\tau)],
\end{array}\right.
$$

where $\Delta_{1}=\omega_{2}-\omega_{1}$ is the frequency mismatch and $\Delta_{2}=\left(\omega_{2}+\omega_{1}\right) / 2$ is the mean natural frequency. Phase-locked solutions of system (1) in terms of variables $\varphi$ and $\phi$ read 


$$
\varphi^{*}=\left[\begin{array}{c}
\arcsin \left[\frac{\Delta_{1}}{2 K \cos (\Omega \tau)}\right] \\
\pi-\arcsin \left[\frac{\Delta_{1}}{2 K \cos (\Omega \tau)}\right]
\end{array}, \phi^{*}=\Omega t+\text { Const },\right.
$$

where the mean frequency $\Omega$ has to be found from the following transcendental equation (cf. [24]),

$$
f_{ \pm}(\Omega):=-\Omega+\Delta_{2} \pm K \sin (\Omega \tau) \sqrt{1-\frac{\Delta_{1}^{2}}{4 K^{2} \cos ^{2}(\Omega \tau)}}=0 \text {. }
$$

In this way, any frequency $\Omega$ found from Eq. (5) defines a single phase-locked solution of the form (4), where the first and the second values in the expression (4) for $\varphi^{*}$ correspond to the sign "+" and "-" in Eq. (5), respectively. Graphically, the solutions $\Omega$ of Eq. (5) are the intersection points of the graph of function $f_{ \pm}$[Fig. 3(a)] with the horizontal axis [Fig. $3(\mathrm{a})$, horizontal solid line $]$. The graph of $f_{ \pm}(\Omega)$ consists of an infinite number of double loops defined on the intervals

$$
\Omega \in\left[\frac{1}{\tau} \pi n-\frac{1}{\tau} \arccos \left(\frac{\Delta_{1}}{2 K}\right), \frac{1}{\tau} \pi n+\frac{1}{\tau} \arccos \left(\frac{\Delta_{1}}{2 K}\right)\right],
$$

and, thus, centered at the points $\Omega_{n}=n \pi / \tau, n \in \mathbb{Z}$.

A detailed analysis of Eq. (5) reveals that an increase of the coupling strength $K$ or delay $\tau$ leads to the emergence of new solutions $\Omega$ and phase-locked states (4) (see also Ref. [24]). The graph of function $f_{ \pm}(\Omega)$ first touches and then intersects zero axis $f_{ \pm}(\Omega)=0$ in two points as $K$ increases [Fig. 3(a)]. The new phase-locked states thus appear in pairs, i.e., via saddle-node bifurcations as the coupling strength gets stronger [Figs. 3(b) and 3(c)]. The numerical evidence suggests that at the saddle-node bifurcations one stable and one unstable solution emerge [Fig. 1(a)]. We address the stability issue of the phase-locked states with the use of the linear stability theory [49]. The characteristic equation for solutions (4) of system (3) reads $\operatorname{det}\left[\lambda I-A-B \mathrm{e}^{-\lambda \tau}\right]=0$, where $I$ is the identity matrix, and $A$ and $B$ are the Jacobi matrices of Eq. (3) with respect to instant and delayed variables, respectively. Taking matrices $A$ and $B$ on the coordinates $\left(\varphi^{*}, \phi^{*}\right)$ of the phase-locked states (4) we obtain that the characteristic equation for the eigenvalues $\lambda$ is independent of time

$$
\begin{aligned}
\lambda^{2}+ & 2 K \cos \left(\varphi^{*}\right) \cos (\Omega \tau) \lambda \mathrm{e}^{-\lambda \tau} \\
& +K^{2} \cos \left(\Omega \tau-\varphi^{*}\right) \cos \left(\Omega \tau+\varphi^{*}\right)\left(\mathrm{e}^{-2 \lambda \tau}-1\right)=0
\end{aligned}
$$

The stability of the phase-locked states can thus be treated as that of fixed points. One can see that $\lambda=0$ is always a solution of Eq. (7). However, this eigenvalue is connected with the invariance of the phase-locked states (4) with respect to a constant shift in variable $\phi$, and, hence, it does not influence the stability of the phase-locked states. The other eigenvalues can be found from Eq. (7) numerically.

Consider, for example, the first pair of the phase-locked states $P$ and $Q$ emerging at the first saddle-node bifurcation as $K$ increases. For the parameter values as in Fig. 3 the
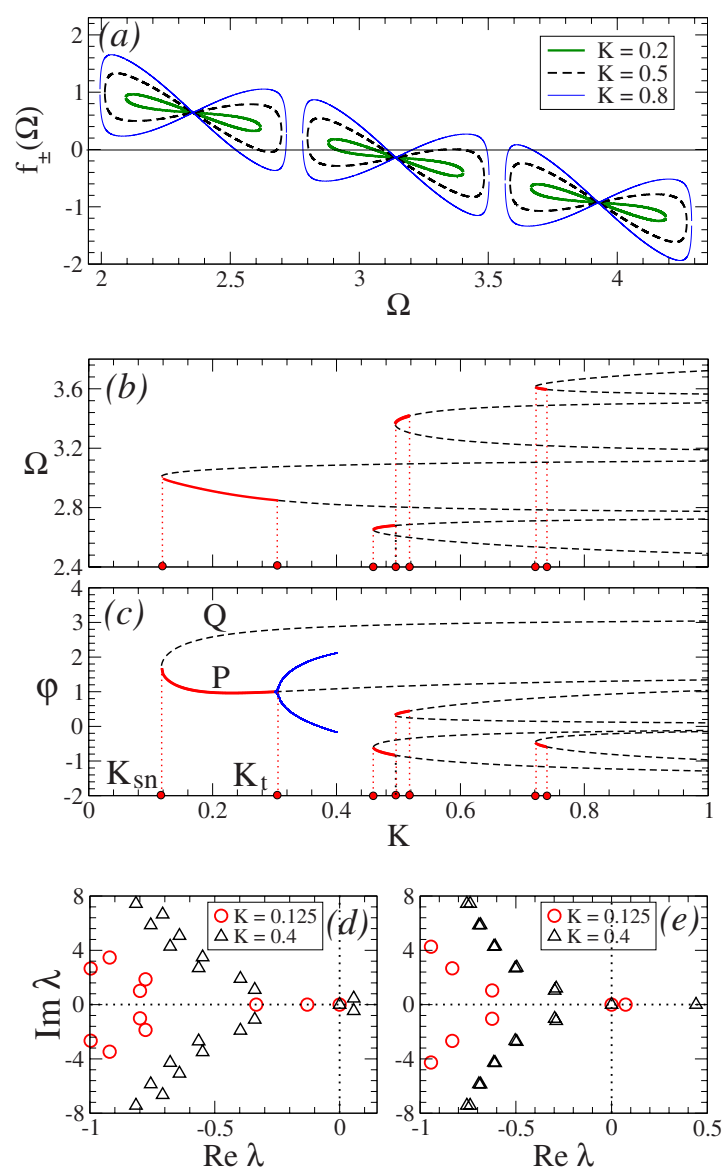

FIG. 3. (Color online) Phase-locked states of system (1). (a) Graph of function $f_{ \pm}(\Omega)$. (5) for three values of $K$ indicated in the legend. (b), (c) Frequencies $\Omega$ and phase differences $\varphi$ of the phaselocked states (4) versus coupling strength $K$. Stable and unstable states are depicted by bold solid red and dashed black curves, respectively. Parameter intervals of stability are indicated by vertical dotted lines. Oscillations of the phase difference, which bifurcate from the stable phase-locked state $P$ at $K=K_{t}$, are illustrated by thin solid blue curves, where the local minima and maxima of the trajectory $\varphi(t)$ are plotted. (d), (e) A few eigenvalues $\lambda$ of the phaselocked states (d) $P$ and (e) $Q$ [see plot (c)] are shown before [red circles] and after [black triangles] the torus bifurcation at $K=K_{t}$. Values of $K$ are indicated in the legends. Other parameters as in Fig. 2.

bifurcation takes place at $K=K_{s n} \approx 0.118$ [Figs. 3(b) and 3(c)]. We found that, as mentioned above, the state $P$ is stable and $Q$ is unstable. Just after the bifurcation, the stable phase-locked state $P$ has three real eigenvalues: one eigenvalue is zero, and the other two are negative [red circles in Fig. 3(d) for $K=0.125$ ]. The remaining (infinitely many) eigenvalues are complex conjugate with negative real parts. The unstable phase-locked state $Q$ is of a saddle-focus type: one eigenvalue is real positive, one eigenvalue is zero, and the others are complex conjugate with negative real parts [red circles Fig. 3(e) for $K=0.125$ ]. As the coupling strength further increases, the two negative real eigenvalues of $P$ meet each other and spread to the complex plane. Next, these eigenvalues cross the imaginary axis at $K=K_{t} \approx 0.305$ and attain positive real parts, and the phase-locked state $P$ loses 

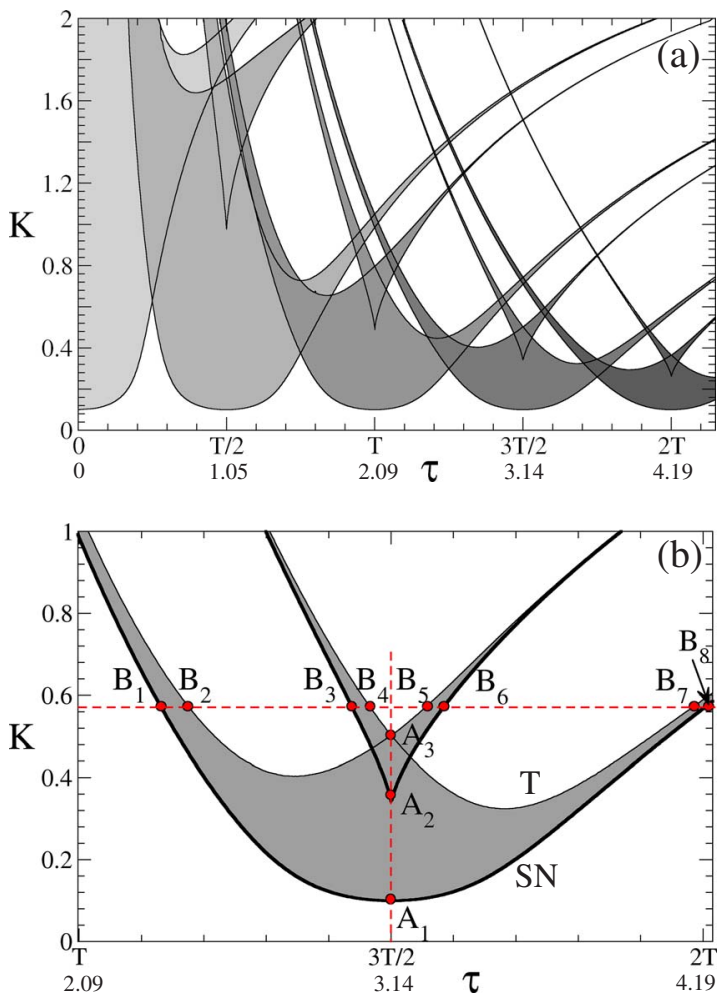

FIG. 4. (Color online) Stability regions of the phase-locked states of system (1) in the $(\tau, K)$-parameter plane. (a) The entire parameter domain consists of an infinite set of swallowlike regions which are shaded by gray of different intensity. (b) Enlarged single swallow stability region in the vicinity of $\tau=3 T / 2$, where $T=2 \pi / \Delta_{2}$ is the mean natural period of system (1). The dashed lines indicate different parameter scans considered in Fig. 5. SN and $\mathrm{T}$ denote saddle-node and torus bifurcations depicted by heavy and thin curves, respectively, which delineate the swallow region. Other parameters as in Fig. 2.

its stability. The eigenvalues of $P$ and $Q$ after the bifurcation are depicted for $K=0.4$ in Figs. 3(d) and 3(e) [black triangles], respectively. After the bifurcation the phase difference $\varphi(t)$ is not constant any longer and periodically oscillates [Fig. 3(c), thin solid blue curves]. Since the phaselocked states can be considered as fixed points, this bifurcation can be interpreted as a supercritical Hopf bifurcation. Above we have, however, seen that for the system of coupled limit-cycle oscillators (2), this is a torus bifurcation, where the second oscillatory mode emerges [Figs. 2(a) and $2(\mathrm{~d})$ ]. We thus call it torus bifurcation below. The same bifurcation scenario is realized for other phase-locked states emerging at consecutive saddle-node bifurcations for larger coupling strength [Figs. 3(b) and 3(c)].

\section{SWALLOW REGIONS}

Stability regions of the phase-locked states for system (1) have an interesting structure in the parameter plane of the delay $\tau$ and the coupling strength $K$ [Fig. 4]. The whole complicated set of parameters $(\tau, K)$ where system (1) has a stable phase-locked state can be classified into infinitely many separate regions which are depicted by gray of differ- ent intensity in Fig. 4(a). A shape of such a single stability region [Fig. 4(b)] strongly resembles that of stability domains of periodic orbits for cubic and quadratic one- and two-dimensional maps called swallows, shrimps, or crossroad areas [36-38]. We however found such objects in the parameter space of a system of two nonlinearly coupled limit-cycle oscillators with delayed feedback. Moreover, in our case these regions are equipped with different bifurcations and exhibit a different organization in the parameter space.

To explore the bifurcations involved in the swallow region, we follow the stability of the phase-locked states as the parameter point enters and leaves the swallow along selected pathways in the two-parameter plane $(\tau, K)$ [Fig. 4(b), dashed lines]. For fixed $\tau=3 T / 2$, where $T=2 \pi / \Delta_{2}$ is the mean natural period of system (1), and varying $K \in(0,0.7)$ [Fig. 4(b), path $A_{1} A_{3}$ ] the evolution of the phaselocked states is illustrated in Figs. 5(a) and 5(b). We found that, for the considered delay $\tau$, system (1) has two phaselocked states at the frequency $\Omega=\Delta_{2}$ if $K \geq \Delta_{1} / 2 \geq 0$. More generally, for the delay $\tau=\frac{T n}{2}, n=0,1,2 \ldots$ the following holds:

$$
f_{ \pm}\left(\Delta_{2}\right)=0 \text {, if and only if }|K| \geq \frac{\left|\Delta_{1}\right|}{2} .
$$

Below we consider the case $\Delta_{1} \geq 0$ and $K \geq 0$ without loss of generality. From Eq. (4) it follows that system (1) has no phase-locked states for $0 \leq K<\Delta_{1} / 2$. Therefore, with increasing $K$ from zero, the very first pair of the phase-locked states (4) $P$ and $Q$ emerges in system (1) via a saddle-node bifurcation at $K=K_{s n}=\Delta_{1} / 2$ and for $\tau=\pi n / \Delta_{2}, n=0,1, \ldots$ [Figs. 5(a) and 5(b) and Fig. 4(b), point $A_{1}$ ]. The $\left(\varphi^{*}, \Omega\right)$-coordinates of the states at the bifurcation are $\varphi^{*}=(-1)^{n} \pi / 2$ and $\Omega=\Delta_{2}$. The frequencies of both phaselocked states $P$ and $Q$ remain the same $\Omega=\Delta_{2}$ for larger $K$, whereas their phase differences $\varphi^{*}$ split from $(-1)^{n} \pi / 2$ and diverge from each other approaching the values 0 and $\pi$ as $K$ increases [Figs. 5(a) and 5(b)]. Let states $P$ and $Q$ have the phase differences $\varphi^{*}=(-1)^{n} \arcsin \left[\Delta_{1} / 2 K\right]$ and $\varphi^{*}=\pi-(-1)^{n} \arcsin \left[\Delta_{1} / 2 K\right]$ from Eq. (4), respectively. Then $P$ is born in a stable manner for even $n=0,2,4, \ldots$, whereas $Q$ is born in a stable manner for odd $n=1,3,5, \ldots$

The above phase-locked states remain stable for $K \in\left(K_{s n}, K_{p f}\right)$ [Figs. 5(a) and 5(b)], where $K_{s n}=\Delta_{1} / 2$, as mentioned above. The other end point of the stability interval can be found

$$
K_{p f}=\sqrt{\frac{1}{\tau^{2}}+\frac{\Delta_{1}^{2}}{4}} .
$$

The value $K=K_{p f}$ defines the $K$-coordinate of the parameter point $A_{2}$ in Fig. 4(b). At the bifurcation at $K=K_{p f}$, a real negative eigenvalue of the stable phase-locked state $P$ or $Q$ crosses zero and becomes positive. The state undergoes a pitchfork bifurcation and loses its stability, and two new stable phase-locked states $P^{\prime}$ and $Q^{\prime}$ emerge [Figs. 5(a) and 5(b)]. $P^{\prime}$ and $Q^{\prime}$ have different frequencies $\Omega_{P^{\prime}}$ and $\Omega_{Q^{\prime}}$ symmetrically located with respect to $\Omega=\Delta_{2}$ [Fig. 5(a)]. In contrast, the $\varphi$-coordinates of $P^{\prime}$ and $Q^{\prime}$ are the same 

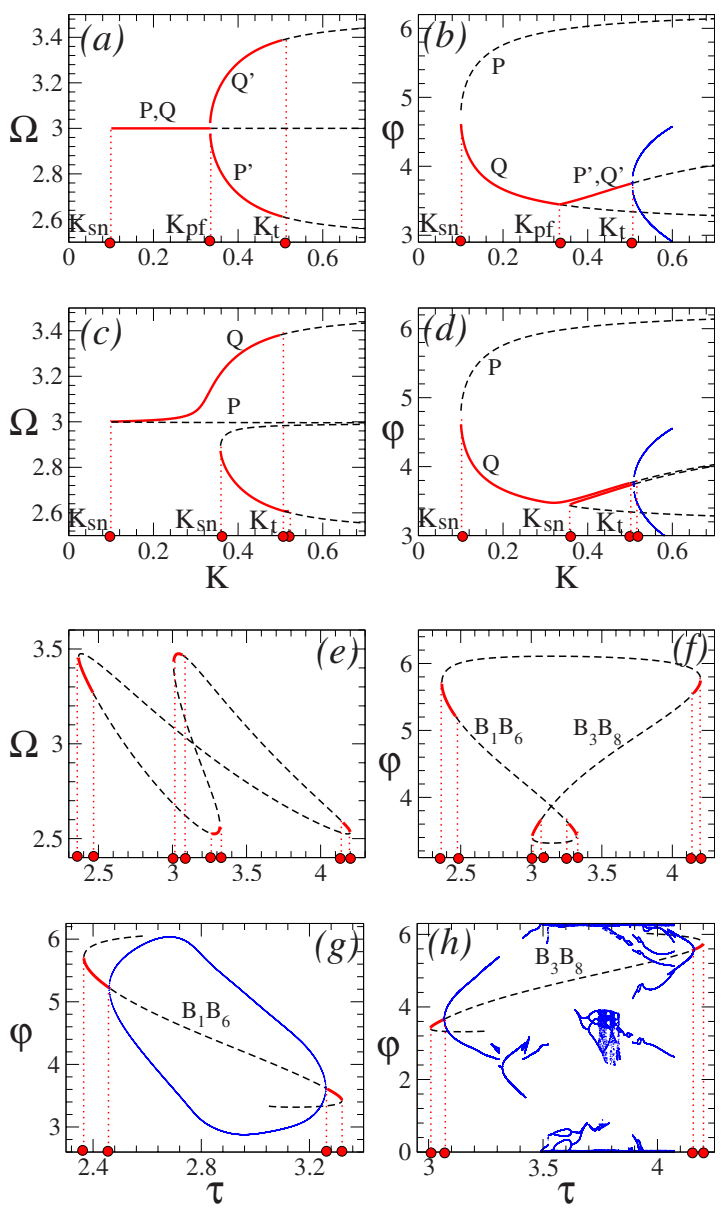

FIG. 5. (Color online) Bifurcations of the phase-locked states of system (1) associated with the swallow stability region [Fig. 4(b)]. One-parameter bifurcation diagrams illustrate the branches of the phase-locked state for fixed delay (a), (b) $\tau=3 T / 2=\pi$ [Fig. 4(b), line $\left.A_{1} A_{3}\right]$, where $T=2 \pi / \Delta_{2}$ is the mean natural period of system (1), and (c), (d) $\tau=3.148$ versus the coupling strength $K$, and (e)-(h) for fixed coupling strength $K=0.575$ [Fig. $4(\mathrm{~b})$, line $B_{1} B_{8}$ ] versus delay $\tau$. (g) and (h) are the enlargements of the branches $B_{1} B_{6}$ and $B_{3} B_{8}$ from (f), respectively. Stable and unstable states are depicted by bold solid red and dashed black curves, respectively. Parameter intervals of stability are indicated by vertical dotted lines. Oscillations of the phase difference $\varphi$ are illustrated by thin solid blue curves, where the local minima and maxima of the trajectory $\varphi(t)$ are plotted. The bifurcation points $K_{s n}, K_{p f}$, and $K_{t}$ denote a saddlenode, pitchfork, and torus bifurcation of the stable phase-locked state and correspond in plots (a) and (b) to the parameter points $A_{1}$, $A_{2}$, and $A_{3}$ in Fig. 4(b), respectively. The bifurcation points in plots (e)-(h) correspond to the parameter points $B_{1}-B_{8}$ in Fig. 4(b). Other parameters as in Fig. 2.

[Fig. 5(b)], which follows from Eq. (4) provided that $\left|\Omega_{P^{\prime}}-\Delta_{2}\right|=\left|\Omega_{Q^{\prime}}-\Delta_{2}\right|$ and $\tau=\pi n / \Delta_{2}$. Depending on the initial conditions, the trajectories of system (1) will be attracted to the stable phase-locked state $P^{\prime}$ or $Q^{\prime}$ exhibiting the same phase difference $\varphi$, but rotating with distinct frequencies $\Omega_{P^{\prime}}$ or $\Omega_{Q^{\prime}}$, respectively.

The phase-locked states $P^{\prime}$ and $Q^{\prime}$ also lose their stability if the coupling strength $K$ in system (1) gets larger [Figs. 5(a) and 5(b)]. Two complex conjugate eigenvalues of $P^{\prime}$ and $Q^{\prime}$ cross the imaginary axis at $K=K_{t}$ and attain positive real parts. After the torus bifurcation at $K=K_{t}$, the phase differences $\varphi(t)$ is not constant any longer, exhibits periodic oscillations, and remains bounded. A regime of periodically modulated phase synchronization is established in system (1). The bifurcation value $K=K_{t}$ defines the $K$-coordinate of the parameter point $A_{3}$ in Fig. 4(b).

For values of the delay slightly away from $\tau=T n / 2$ considered above [Figs. 5(a) and 5(b)] the bifurcation scenario changes. The pitchfork bifurcation is replaced by an additional saddle-node bifurcation [Figs. 5(c) and 5(d)]. The other bifurcations are preserved, e.g., the phase-locked states lose their stability via a torus bifurcation and the phase difference starts to oscillate as the coupling strength in system (1) increases, see also Fig. 1(a).

Finally, we fix the coupling $K=0.575$ and vary the delay $\tau \in(2.2,4.2)$ [Fig. 4(b), line $B_{1} B_{8}$ ]. If the parameter point enters the swallow through the outer border of the left "wing" [Fig. 4(b), point $B_{1}$ ] as the delay $\tau$ increases, two phase-locked states emerge via a saddle-node bifurcation. One of them is unstable and the other one is stable [Figs. $5(\mathrm{e})-5(\mathrm{~g})]$. The stable state then undergoes a torus bifurcation and destabilizes if the scanning parameter point leaves the left wing of the swallow through its inner border [Fig. 4(b), point $B_{2}$ ]. This branch of solution continues up to the parameter point $B_{6}$ [Fig. 4(b)]. There, the considered phaselocked state, getting stable via an inverse torus bifurcation at the point $B_{5}$, undergoes a saddle-node bifurcation and disappears [Figs. $5(\mathrm{e})-5(\mathrm{~g})]$. In parallel, another branch of stable solution emerges via a saddle-node bifurcation if the parameter point enters the left "tail" of the swallow at the parameter point $B_{3}$ [Fig. 4(b)]. The stable phase-locked state then undergoes the same sequence of bifurcations as that of the branch $B_{1} B_{6}$ considered above [Figs. 5(e), 5(f), and 5(h)]: It loses and again attains its stability via a direct and inverse torus bifurcation at the bifurcation points $B_{4}$ and $B_{7}$ [Fig. 4(b)], respectively, and finally disappears via a saddle-node bifurcation if the scanning parameter point leaves the swallow through the outer border of the right wing at the point $B_{8}$ [Fig. 4(b)]. Therefore, two branches of the phase-locked solutions $B_{1} B_{6}$ and $B_{3} B_{8}$ simultaneously exist [Figs. 5(e)-5(h)] and connect the left wing of the swallow with its right tail and, correspondingly, the right wing of the swallow with its left tail [Fig. 4(b)]. In such a way four phase-locked states exist between the tails of the swallow, and two phase-locked states exist between the wings of the swallow [Figs. $5(\mathrm{e})-5(\mathrm{~h})]$.

\section{SYNCHRONIZED DYNAMICS}

As mentioned above, if the parameter point leaves the swallow through its inner border [Fig. 4(b), thin curve denoted by "T"], a stable phase-locked state loses its stability via a torus bifurcation, and a stable oscillatory dynamics of the phase difference $\varphi(t)$ emerges in system (1). The latter can then follow a rather complicated sequence of bifurcations as parameters vary between the wings of the swallow. For example, the oscillations of the phase difference can remain bounded and regular along the entire parameter path- 


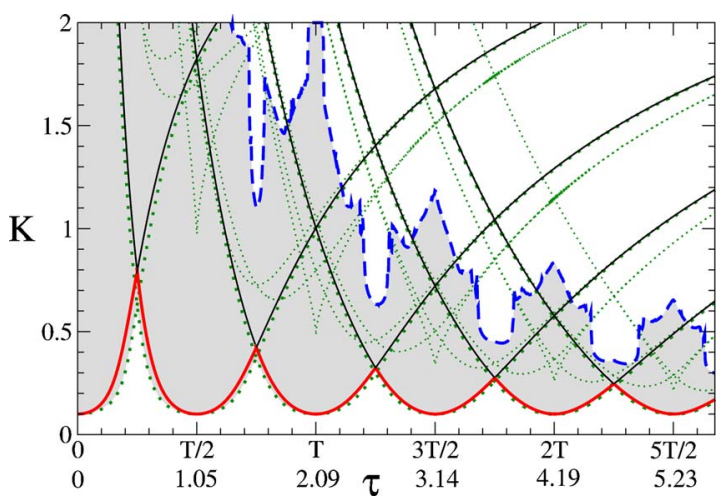

FIG. 6. (Color online) Synchronization region (shaded by gray) of the two phase oscillators with delayed feedback (1). The swallow regions of stable phase-locked states are delineated by dotted green curves. The analytical approximation (10) of the lower borders of the swallows are depicted by solid bold red and thin black curves. Parameter thresholds of synchronization and desynchronization onsets in system (1) with respect to the increasing coupling strength $K$ are indicated by bold solid red and bold dashed blue curves, respectively. Other parameters as in Fig. 2.

way from their emergence up to the disappearance [Fig. 5(g)]. On the other hand, the limit cycle of the phase difference can develop to a chaotic behavior for other parameter values [Fig. 5(h), see also Fig. 1(a)]. Moreover, the phase difference can also get unbounded, which indicates an onset of desynchronization of oscillators (1). For example, the phase difference $\varphi$ unlimitedly grows for the delay parameter $\tau \in(3.53,3.83)$ [Fig. 5(h)] along the parameter scan $B_{1} B_{8}$ [Fig. 4(b)].

Synchronized dynamics of oscillators (1) strongly relates to the swallow stability regions of the phase-locked states [Fig. 4]. For weak coupling the oscillators are not synchronized because of different natural frequencies $\omega_{j}$. They synchronize for larger coupling strength, if the parameter point enters one of the swallow region through its lower border [Fig. 6, bold solid red curve], and the oscillators' phases get locked to each other. The smallest coupling strength, at which the oscillators can synchronize, $K=\Delta_{1} / 2$ for $\tau=T n / 2$, $n=0,1, \ldots$, where $T=2 \pi / \Delta_{2}$ is the mean natural period. Such values of the delay $\tau$ define the centers of the swallow stability regions (Fig. 4). Then the phase-locked synchronization is destroyed via a torus bifurcation as the coupling increases and the parameter point leaves the swallow through its upper border [Figs. 4 and 5]. The phase difference starts to periodically oscillate and still remains bounded, i.e., the oscillators are still synchronized. The limit cycle for the phase difference in turn undergoes further bifurcations and may develop to a chaotic attractor for larger coupling strength [Figs. 1 and 5]. Finally, the oscillators desynchronize. We evaluate this bifurcation moment in the $(\tau, K)$-parameter plane [Fig. 6, bold dashed blue curve]. The bifurcation curve of desynchronization onset follows the swallow structure in the parameter space. The values of the delay $\tau$ at the centers of the swallows or close to them promote synchronization, whereas synchronization is less pronounced for the values of the delay between the swallows [Fig. 6]. We note that, even above the desynchronization pa- rameter threshold [Fig. 6, bold dashed blue curve], parameter windows of synchronization can be found, which are connected, e.g., with wings and tails of other swallows.

The lower boundaries of the swallows can be found analytically. For this we look for parameter values where Eq. (5) attains solutions. This takes place when the extrema of the graph of the function $f_{ \pm}(\Omega)$ touch the horizontal axis [Fig. $3(\mathrm{a})]$. Then the additional condition to Eq. (5) reads $f_{ \pm}^{\prime}(\Omega)=0$. Solving this system we obtain the equation for the evaluation of the lower boundary of the swallows

$$
4 K^{4} \cos ^{8}(\Omega \tau)-4 K^{4} \cos ^{6}(\Omega \tau)-K^{2} \cos ^{4}(\Omega \tau) \Delta_{1}^{2}+\Delta_{1}^{2} / 4=0 .
$$

It can be solved analytically, but the solutions are too cumbersome to present them here. Instead, we approximate the condition $f_{ \pm}^{\prime}(\Omega)=0$ by $g_{ \pm}^{\prime}(\Omega)=0$, where the function $g_{ \pm}(\Omega)=f_{ \pm}(\Omega)+\Omega-\Delta_{2}$ is obtained by aligning the graph of the function $f_{ \pm}(\Omega)$ [Fig. 3(a)] along the horizontal zero axis [Fig. 3(a), horizontal solid line]. It can be shown that the solutions of the approximate condition $g_{ \pm}^{\prime}(\Omega)=0$,

$$
\Omega_{g}= \pm \frac{1}{\tau} \arccos \left(\sqrt{\frac{\Delta_{1}}{2 K}}\right)+\frac{1}{\tau} \pi n
$$

only slightly deviate from the exact solutions $\Omega_{f}$, and their mismatch can be estimated as follows:

$$
\left|\Omega_{f}-\Omega_{g}\right| \leq \frac{1}{\tau}\left|\arccos \left(\frac{\Delta_{1}}{2 K}\right)-\arccos \left(\sqrt{\frac{\Delta_{1}}{2 K}}\right)\right| .
$$

This follows from the observation that the exact solution $\Omega_{f}$ of interest is located in the $\Omega$-interval bounded by the corresponding approximate solution $\Omega_{g}$ from one side and by the end point nearest to $\Omega_{g}$ in the interval of the existence (6) from the other side. Substituting the solutions $\Omega_{g}$ into Eq. (5) we obtain a compact expression for the lower boundary of the swallows

$$
\tau=\left( \pm \operatorname{acrcos}\left(\sqrt{\frac{\Delta_{1}}{2 K}}\right)-\pi n\right) /\left( \pm \frac{\Delta_{1}-2 K}{2}-\Delta_{2}\right),
$$

$n=0,1,2, \ldots$

The above expression (10) for the lower boundaries of the swallows [Fig. 6, solid bold red and thin black curves] well approximates the results of numerical calculations [Fig. 6, green dots]. This approximation improves for large delay $\tau$ and coupling strength $K$. The minimal values of $K$ from Eq. (10) versus the delay $\tau$ and index $n$ give the lower parameter boundary of the stable synchronized dynamics in system (1) [Fig. 6, bold solid red curves].

\section{DISCUSSION}

In this paper, we studied phase-locked states of two nonlinearly coupled limit-cycle oscillators with delayed feedback. The phase dynamics of the oscillators is well approximated by a system of two coupled phase oscillators with delayed feedback. Such a system exhibits a variety of synchronized regimes ranging from phase locking to periodically modulated and chaotic phase synchronization. Interest- 
ingly, the oscillators desynchronize if the coupling between them becomes large. Although linearly coupled complex systems, for example, $x$-coupled Rössler oscillators [50] can desynchronize as coupling strength increases, this is in contrast to other systems of linearly coupled phase oscillators [1,2], even with a time delay in their coupling [22-24,26,27].

The stability regions of the phase-locked states in the parameter space are found to have the form of swallows (also called shrimps or crossroad areas) previously reported for nonlinear time-discrete maps [34-38] and many other systems [6,39-46]. We however found that, in the considered system, the borders of the swallow regions are delineated by the bifurcation curves of a saddle-node bifurcation and a torus bifurcation. The periodic orbits of the phase-locked solutions do not undergo a period-doubling bifurcation, and, thus, the swallows do not form a hierarchical nesting structure in the parameter space contrary to the previous findings $[6,35,37,41,47,48]$. We therefore report on a different bifurcation scenario associated with the swallows or shrimps, which are commonly accepted to be universal structures in the parameter space of very different dynamical systems.

The reported swallow stability regions play an important role in the onset of synchronization and constitute the lower coupling threshold of the synchronization domain. We analytically approximate this synchronization threshold. The bifurcation curve has an interesting resonancelike structure with respect to the delay similar to that found for the Kuramoto model with delay [22] or mechanical oscillatorpendulum system modeled by neutral delay differential equa- tions [51], see also Ref. [24]. Such a bifurcation curve might be connected to a periodic reappearance (with respect to the delay) of periodic solutions studied for Duffing and StuartLandau oscillators with delay [52].

The amplitude and phase dynamics observed for the two nonlinearly coupled oscillators (2), where the increasing coupling strength causes a transition from a phase-locked state characterized by constant amplitudes to a quasiperiodic regime, seems to be a generic phenomenon. An onset of a quasiperiodic dynamics has been found for two linearly coupled semiconductor lasers interacting with time delay [53]. Similar transitions for the amplitude and phase dynamics have been studied for a large array of semiconductor lasers [11], which has been modeled by a generalized Kuramoto system with nonlinear delayed coupling. Such a coupling leads to the onset of quasiperiodic and even chaotic synchronization among lasers as the coupling strength increases, which we also observed in our system of two oscillators. The results obtained in this paper contribute to the classification of the parameter space of interacting systems where the coupling has a complex nonlinear form including time delay.

Finally we note that, as mentioned in the introduction, the nonlinear coupling with delayed feedback considered for the two limit-cycle oscillators (2) has been used to develop a nonlinear delayed feedback method [30-33] for the control of synchronization in oscillatory neuronal ensembles, aiming at applications to medicine.
[1] Y. Kuramoto, Chemical oscillations, Waves, and Turbulence (Springer, Berlin; Heidelberg; New York, 1984).

[2] A. Pikovsky, M. Rosenblum, and J. Kurths, Synchronization, a Universal Concept in Nonlinear Sciences (Cambridge University Press, Cambridge, England, 2001).

[3] A. Balanov, N. Janson, D. Postnov, and O. Sosnovtseva, Synchronization: from simple to complex (Springer, New York, 2009).

[4] D. C. Michaels, E. P. Matyas, and J. Jalife, Circ. Res. 61, 704 (1987).

[5] E. M. Izhikevich, SIAM Rev. 43, 315 (2001).

[6] L. Glass, Nature (London) 410, 277 (2001).

[7] H. Kori and A. S. Mikhailov, Phys. Rev. Lett. 93, 254101 (2004).

[8] K. Wiesenfeld, P. Colet, and S. H. Strogatz, Phys. Rev. Lett. 76, 404 (1996).

[9] I. Z. Kiss, W. Wang, and J. L. Hudson, J. Phys. Chem. B 103, 11433 (1999).

[10] S. H. Strogatz, D. M. Abrams, A. McRobie, B. Eckhardt, and E. Ott, Nature (London) 438, 43 (2005).

[11] G. Kozyreff, A. G. Vladimirov, and P. Mandel, Phys. Rev. Lett. 85, 3809 (2000).

[12] T. Heil, I. Fischer, W. Elsasser, J. Mulet, and C. R. Mirasso, Phys. Rev. Lett. 86, 795 (2001).

[13] W. Wang, I. Z. Kiss, and J. L. Hudson, Ind. Eng. Chem. Res. 41, 330 (2002).
[14] B. Dorizzi and B. Grammaticos, Phys. Rev. A 44, 6958 (1991).

[15] W. Gerstner, Phys. Rev. Lett. 76, 1755 (1996).

[16] M. Yoshioka and M. Shiino, Phys. Rev. E 58, 3628 (1998).

[17] S. M. Crook, G. B. Ermentrout, M. C. Vanier, and J. M. Bower, J. Comput. Neurosci. 4, 161 (1997).

[18] U. Ernst, K. Pawelzik, and T. Geisel, Phys. Rev. Lett. 74, 1570 (1995).

[19] J. Faro and S. Velasco, Physica D 110, 313 (1997).

[20] A. Beuter, J. Belair, C. Labrie, and J. Belair, Bull. Math. Biol. 55, 525 (1993).

[21] P. Tass, J. Kurths, M. G. Rosenblum, G. Guasti, and H. Hefter, Phys. Rev. E 54, R2224 (1996).

[22] M. K. Stephen Yeung and S. H. Strogatz, Phys. Rev. Lett. 82, 648 (1999).

[23] M. G. Rosenblum and A. S. Pikovsky, Phys. Rev. Lett. 92, 114102 (2004).

[24] H. G. Schuster and P. Wagner, Prog. Theor. Phys. 81, 939 (1989).

[25] U. Ernst, K. Pawelzik, and T. Geisel, Phys. Rev. E 57, 2150 (1998).

[26] M. Y. Choi, H. J. Kim, D. Kim, and H. Hong, Phys. Rev. E 61, 371 (2000).

[27] B. Lysyansky, Y. L. Maistrenko, and P. A. Tass, Int. J. Bifurcation Chaos Appl. Sci. Eng. 18, 1791 (2008).

[28] V. Krachkovskyi, O. V. Popovych, and P. A. Tass, Phys. Rev. E 
73, 066220 (2006).

[29] O. V. Popovych, V. Krachkovskyi, and P. A. Tass, Int. J. Bif. Chaos 17, 2517 (2007).

[30] O. V. Popovych, C. Hauptmann, and P. A. Tass, Phys. Rev. Lett. 94, 164102 (2005).

[31] O. V. Popovych, C. Hauptmann, and P. A. Tass, Biol. Cybern. 95, 69 (2006).

[32] O. V. Popovych, C. Hauptmann, and P. A. Tass, J. Biol. Phys. 34, 267 (2008).

[33] O. V. Popovych and P. A. Tass, Phys. Rev. E 82, 026204 (2010).

[34] H. El Hamouly and C. Mira, C. R. Acad. Sci. (Paris) 294, 387 (1982).

[35] J. Ringland, N. Issa, and M. Schell, Phys. Rev. A 41, 4223 (1990).

[36] J. Milnor, Exp. Math. 1, 5 (1992).

[37] J. A. C. Gallas, Physica A 202, 196 (1994).

[38] R. Allam and C. Mira, Int. J. Bifurcation Chaos Appl. Sci. Eng. 3, 429 (1993).

[39] C. Scheffczyk, U. Parlitz, T. Kurz, W. Knop, and W. Lauterborn, Phys. Rev. A 43, 6495 (1991).

[40] C. Bonatto, J. C. Garreau, and J. A. C. Gallas, Phys. Rev. Lett. 95, 143905 (2005).

[41] Y. Zou, M. Thiel, M. C. Romano, J. Kurths, and Q. Bi, Int. J.
Bifurcation Chaos Appl. Sci. Eng. 16, 3567 (2006).

[42] V. Castro, M. Monti, W. B. Pardo, J. A. Walkenstein, and E. Rosa, Int. J. Bifurcation Chaos Appl. Sci. Eng. 17, 965 (2007).

[43] J. G. Freire, R. J. Field, and J. A. C. Gallas, J. Chem. Phys. 131, 044105 (2009).

[44] S. L. T. de Souza, I. L. Caldas, and R. L. Viana, Math. Probl. Eng. 2009, 290356 (2009).

[45] R. Perez and L. Glass, Phys. Lett. A 90, 441 (1982).

[46] M. Schell, S. Fraser, and R. Kapral, Phys. Rev. A 28, 373 (1983).

[47] J. Bélair and L. Glass, Physica D 16, 143 (1985).

[48] B. R. Hunt, J. A. C. Gallas, C. Grebogi, J. A. Yorke, and H. Kocak, Physica D 129, 35 (1999).

[49] R. Bellman and K. L. Cooke, Differential-Difference Equations (Academic Press, New York, 1963).

[50] L. M. Pecora, T. L. Carroll, G. A. Johnson, D. J. Mar, and J. F. Heagy, Chaos 7, 520 (1997).

[51] Y. N. Kyrychko, K. B. Blyuss, A. Gonzalez-buelga, S. J. Hogan, and D. J. Wagg, Proc. R. Soc. London, Ser. A 462, 1271 (2006).

[52] S. Yanchuk and P. Perlikowski, Phys. Rev. E 79, 046221 (2009).

[53] A. Hohl, A. Gavrielides, T. Erneux, and V. Kovanis, Phys. Rev. Lett. 78, 4745 (1997). 